Article

\title{
Accelerated Aging of the Traditional Greek Distillate Tsipouro Using Wooden Chips. Part I: Effect of Static Maceration vs. Ultrasonication on the Polyphenol Extraction and Antioxidant Activity
}

\author{
Theodora Taloumi and Dimitris P. Makris * \\ Department of Food Science \& Nutrition, University of the Aegean, Mitr. Ioakim Street, \\ Myrina 81400, Lemnos, Greece; fns11094@fns.aegean.gr \\ * Correspondence: dmakris@aegean.gr; Tel.: +30-2254-083-114 \\ Academic Editor: James Harbertson \\ Received: 29 November 2016; Accepted: 7 January 2017; Published: 17 January 2017
}

\begin{abstract}
The Greek traditional grape marc distillate Tsipouro was subjected to accelerated aging, using wooden chips from acacia, cherry, chestnut and oak wood. The processes included treatments under static maceration and ultrasonication and the evolution of the total polyphenol concentration was monitored over a period of 30 days. During this period, leaching of polyphenols from the chips into the distillate was found to obey first-order kinetics, but no statistical differences were shown between the two treatments regarding the enrichment of the liquid in polyphenolic substances. The determination of the antioxidant activity demonstrated that aging with chestnut chips may provide Tsipouro with particularly strong radical scavenging and reducing effects, highlighting its importance as a material that could be used to turn distillates into foods with functional properties.
\end{abstract}

Keywords: aging; antioxidans; distillate; kinetics; polyphenols; Tsipouro

\section{Introduction}

Aging is an important process affecting the quality of distilled beverages and it has become a common practice for several distilled drinks, such as whiskies and brandies, to mature, usually in oak casks [1]. The aging period in wooden casks is considered fundamental in contributing to the organoleptic characteristics of distillates, but there is an increasing trend in the implementation of alternative techniques of aging. This is because the use of casks entails a high associated cost, the aging time is long and very large floor space is required. The most popular method replacing traditional cask aging is the use of wooden fragments, collectively known as chips, which are added in wines and distillates to achieve the extraction of non-volatile and volatile compounds, which largely define the quality of the finished product. Furthermore, wooden chip-aided aging is of considerably lower cost, while the aging period is dramatically shortened. Oak chips of various sizes and toasting levels are now commercially available and are increasingly being use in this way [2], given the authorization by the EU (Council Regulation (EC) No. 2165/2005 of 20 December 2005).

There have been previous attempts to use alternative means of accelerating the aging of distillates, by deploying methods including ultrasonication, which was claimed to simulate phenomena associated with aging [3]. However, only recently, a novel approach for wine aging, implementing ultrasounds combined with oak chips, has been proposed [4]. Ultrasounds may induce the acceleration of solid material diffusion into the liquid through acoustic cavitation (formation, growth and implosive collapse of bubbles). Ultrasonic power is known to provoke voids in a liquid, characterized as cavitation bubbles, which are responsive to the ultrasonic effect. During ultrasonication, these bubbles are able to grow up to a critical point, beyond which they collapse, releasing large amounts of energy. The combination of 
the high temperature/high pressure involved in such a process disrupts the solid particle integrity, resulting in the release of the solute in the liquid phase [5].

On such grounds, ultrasonication could assist in the release of material from wooden chips, thus significantly enhancing the enrichment of a distillate in various substances, and more specifically polyphenols. Polyphenols are a highly versatile group of secondary metabolites, which possess a variety of biological properties, beneficial to human health [6]. Hence, any treatment of distillates for the purpose of fortification with such functional ingredients would significantly add value to these beverages, turning them into foods with functional (antioxidant) properties. Tsipouro is a traditional Greek distillate, produced by the distillation of the residual from winemaking, mostly the marc. Similar spirits have equivalent appellations, such as Italian "grappa", and French "eau-de-vie de marc". Tsipouro production includes the selection of the grape variety, the fermentation of the grape marc and then distillation, and for some viticulture regions Tsipouro has been recognized as a product of protected geographical designations (Annex III, Regulation (EC) No. 110/2008). Tsipouro has been extensively studied with regard to volatile constituents [7-9] and the impact of various types of packaging [10]. More recent studies successfully performed discrimination of Tsipouro samples from various regions of Greece, based on NMR metabolite profiling [11,12]. However, since aged Tsipouro has rather recently been launched in the Greek market, there are no data on the polyphenolic load and antioxidant properties of such commodities. Given the importance of aging not only to the sensory properties of distillates, but also to their functionality, information regarding the effect of traditional (barrel) or accelerated (chips) aging is imminent. On such a basis, this project was undertaken with the aim of (i) tracing the kinetics of polyphenol release into Tsipouro using wooden chips from various botanical species; (ii) assessing the effect of ultrasonication on this process; (iii) identifying wood species that could highly enrich Tsipouro in polyphenolic ingredients; and (iv) estimating the impact of accelerated aging on the antioxidant properties of the distillate.

\section{Materials and Methods}

\subsection{Chemicals}

Ascorbic acid, 2,4,6-tripyridyl-s-triazine (TPTZ), Folin-Ciocalteu reagent, gallic acid, rutin (quercetin 3-O-rutinoside) and 2,2-diphenyl-picrylhydrazyl $\left(\mathrm{DPPH}^{\bullet}\right.$ ) stable radical were from Sigma Chemical Co. (St. Louis, MO, USA). Absolute ethanol was from Fisher Scientific (Bridgewater, NJ, USA). Sodium acetate and aluminium chloride were from Penta (Prague, Czeck Republic).

\subsection{Wooden Chips}

The chips used in this study were from four different botanical species, namely white oak (Quercus alba), false acacia (Robinia pseudoacacia), cherry (Prunus avium) and chestnut (Castanea sativa) and were kindly donated by the Department of Wood and Furniture Design and Technology (T.E.I. of Thessaly, Karditsa). The chips underwent no thermal treatment prior to use (non-toasted) and they had average dimensions $1.0 \mathrm{~cm} \times 1.0 \mathrm{~cm} \times 0.5 \mathrm{~cm}$ and average weight approximately $0.250 \mathrm{~g}$. The material was placed in sealed plastic tubes, in a dark and dry chamber, until used.

\subsection{Distillate}

Tsipouro used was made from marc originating from the Greek native Vitis vinifera variety Muscat of Lemnos. The beverage was kindly provided by a local distillery (Myrina, Lemnos) and it had alcohol content of $49 \%(v / v)$.

\subsection{Aging Treatments}

Aliquots of $250 \mathrm{~mL}$ Tsipouro were placed in $500 \mathrm{~mL}$ screw-cap glass bottles and wooden chips were added to provide an approximate content of $4.5 \mathrm{~g} \cdot \mathrm{L}^{-1}$. The bottles were sealed and stored at room temperature $\left(28 \pm 1{ }^{\circ} \mathrm{C}\right)$, over a period of 30 days. For the ultrasonication treatment, bottles were 
immersed in a sonication bath (Elma P70, Singen, Germany) and sonicated for $5 \mathrm{~min}$ at $72 \mathrm{~h}$ intervals. Sonication frequency was set at $37 \mathrm{kHz}$, sonication power at $200 \mathrm{~W}$ and acoustic energy density at $50 \mathrm{~W} \cdot \mathrm{L}^{-1}$.

\subsection{Sampling and Determinations}

Sampling was accomplished at $72 \mathrm{~h}$ intervals, following a short (2-3 s), mild manual shaking of the bottles, to ensure obtaining of homogeneous solutions. Total polyphenol concentration $\left(C_{\mathrm{TP}}\right)$ was determined with the Folin-Ciocalteu methodology [13] and expressed as gallic acid equivalents (mg.GAE. $\mathrm{L}^{-1}$ ). Total flavonoid concentration $\left(C_{\mathrm{TFn}}\right)$ was estimated with the $\mathrm{AlCl}_{3}$ reagent, as described elsewhere [14] and expressed as rutin equivalents (mg RtE. $\left.\mathrm{L}^{-1}\right)$. Reducing power $\left(\mathrm{P}_{\mathrm{R}}\right)$ was estimated using the TPTZ methodology [15] and results were expressed as ascorbic acid equivalents (mM AAE). The antiradical activity $\left(\mathrm{A}_{\mathrm{AR}}\right)$ was measured with the DPPH probe, as described previously [16].

\subsection{Statistical Analysis}

Treatments were repeated at least twice and all determinations at least in triplicate. Values reported are averages and standard deviations. Correlations and value distributions were determined using linear and non-linear regression and distribution analysis, respectively, at least at a 95\% significance level. Statistics was performed with JMP ${ }^{\circledR} 10$ and SigmaPlot ${ }^{\circledR} 12.0$.

\section{Results and Discussion}

\subsection{Polyphenol Extraction Kinetics}

A previous examination of wine-like model solutions demonstrated that the addition of chips from acacia, cherry and oak enriched the liquid medium in polyphenolic substances, but significant differences in the extraction kinetics were observed among chips [13]. This finding was attributed to differences in the structure of the wood originating from various botanic species, but also to the polyphenolic load of each wood. Furthermore, earlier investigations showed that the ethanol concentration is a key factor in defining the kinetics of polyphenol extraction from wooden chips in liquid model matrices [17]. In this study, the liquid matrix used was the distillate Tsipouro and treatments included the use of wooden chips from four different botanic species. Tsipouro was subjected to accelerated aging by employing the commonly used static maceration (SM). Furthermore, as recent reports demonstrated an enhancement of polyphenol release from oak chips into model wines upon treatment with ultrasonication (US) [18], this technique was also employed to identify conditions that could facilitate polyphenol extraction.

The use of chips from any botanic species resulted in coloration of the distillate (data not shown); however, visual inspection did not show distinguishable differences between samples that had undergone static maceration and ultrasonication, although differences among chips from the various woods were evident. Thus, to obtain insight into the polyphenol extraction kinetics and the polyphenolic composition, the course of polyphenol leaching from the wooden chips into the distillate was monitored for 30 days, by determining $C_{\mathrm{TP}}$ at regular intervals, as shown in Figure 1 . Data processing indicated that the best-fitting model was first-order kinetics, described as follows [19]:

$$
C_{\mathrm{TP}(t)}=C_{\mathrm{TP}(s)}\left(1-e^{-k t}\right)
$$

where $C_{\operatorname{TP}(t)}$ is the total polyphenol concentration in the distillate at time $t, C_{\operatorname{TP}(s)}$ is the total polyphenol concentration in the distillate at saturation (equilibrium) and $k$ is the apparent first-order extraction rate constant. $C_{\mathrm{TP}(s)}$ and $k$ were estimated by performing non-linear regression, using SigmaPlot ${ }^{\mathrm{TM}}$ 12.0, and the values are analytically presented in Table 1 . The fact that polyphenol release obeyed first-order kinetics contrasted findings from previous studies on model wines [13,18], which showed polyphenol release to match second-order kinetics. Another empirical model proposed earlier 
suggested polyphenol extraction from oak chips into hydroalcoholic media to be dependent on the ethanol concentration [17]. Since Tsipouro has a considerably higher alcohol content, then the modification in the kinetic pattern would be reasonable.
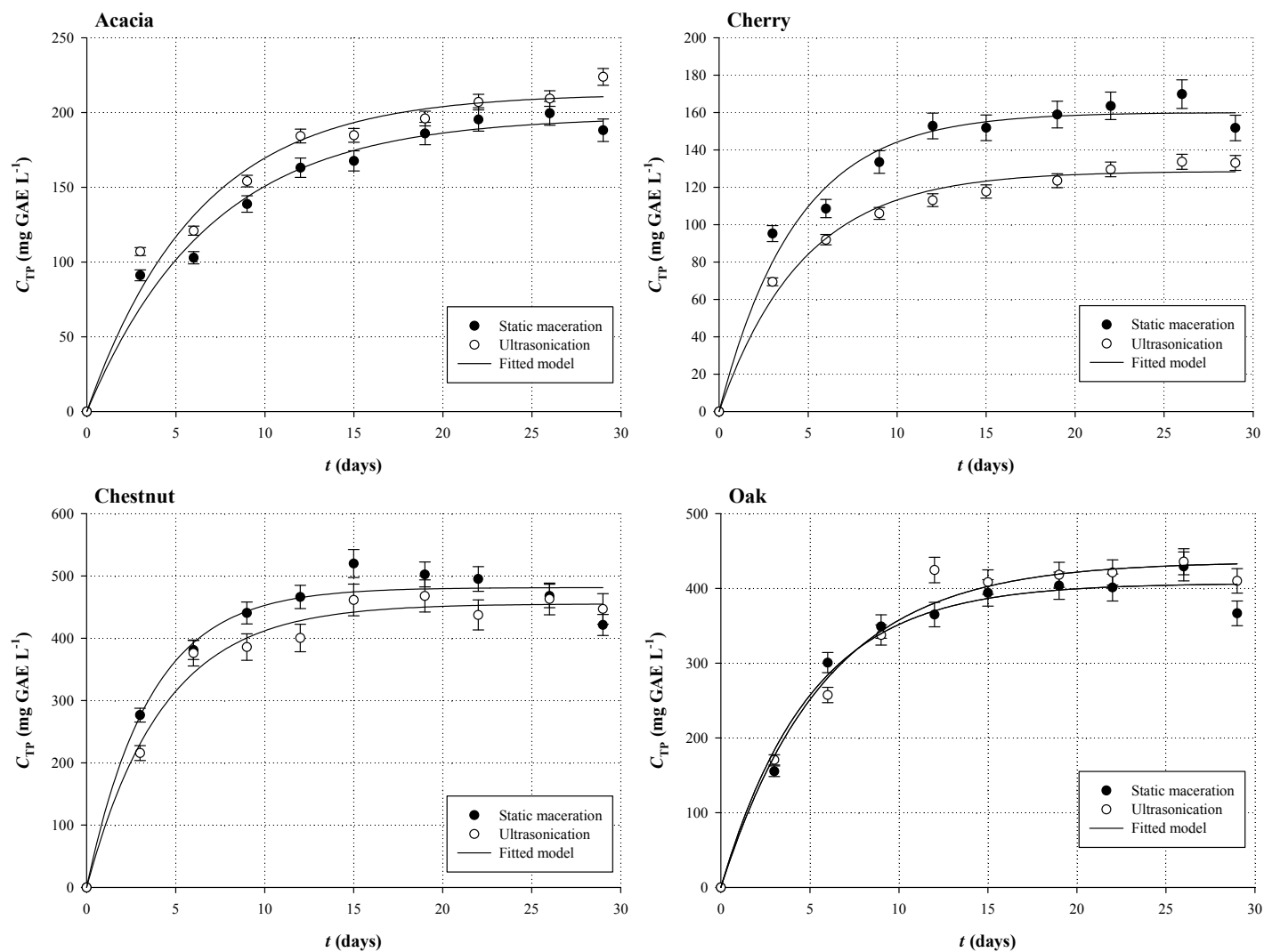

Figure 1. Time course of polyphenol release from wooden chips into Tsipouro, under SM (static maceration) and US (ultrasonication). Treatments were carried out over a period of 30 days.

Table 1. Kinetic parameters estimated for accelerating aging of Tsipouro using wooden chips from various botanical species, under SM (static maceration) and US (ultrasonication). Treatments were carried out over a period of 30 days.

\begin{tabular}{|c|c|c|}
\hline \multirow{2}{*}{ Sample } & \multicolumn{2}{|c|}{ Kinetic Parameters } \\
\hline & $k$ (days $\left.^{-1}\right)$ & $C_{\mathrm{TP}(s)}\left(\mathrm{mg} \cdot \mathrm{GAE} \cdot \mathrm{L}^{-1}\right)$ \\
\hline \multicolumn{3}{|c|}{ Acacia } \\
\hline SM & 0.145 & 197.24 \\
\hline US & 0.160 & 212.71 \\
\hline \multicolumn{3}{|c|}{ Cherry } \\
\hline SM & 0.231 & 160.10 \\
\hline US & 0.212 & 128.63 \\
\hline \multicolumn{3}{|c|}{ Chestnut } \\
\hline SM & 0.282 & 481.47 \\
\hline US & 0.235 & 455.39 \\
\hline \multicolumn{3}{|c|}{ Oak } \\
\hline SM & 0.200 & 406.96 \\
\hline US & 0.172 & 435.84 \\
\hline
\end{tabular}


For the aliquots of Tsipouro treated with cherry, chestnut and oak chips, it was found that $\mathrm{SM}$ resulted in faster extraction, yielding higher $k$. By contrast, US facilitated extraction only from acacia chips. However, US enhanced polyphenol release from oak chips, and $C_{\mathrm{TP}(s)}$ determined in the ultrasonicated sample was $6.6 \%$ higher than that in the sample treated with SM. Likewise, in the sample aged with acacia chips, US provoked a 7.3\% higher enrichment in total polyphenols. Nevertheless, in both cases, the differences were statistically insignificant $(p<0.05)$. The same was also observed for samples treated with chestnut chips, where the difference between SM and US was almost 5.5\%. On the other hand, the sample aged under SM was approximately by $19.7 \%$ richer in total polyphenols compared with that which underwent US, in the case where cherry chips were used. This outcome is rather paradoxical, considering that US is an effective means of enhancing the extractability of substances from solids into the liquid matrix, by providing the appropriate dissipation energy, which is required to disrupt the integrity of the solids [20]. Therefore, it would normally be anticipated that US would increase polyphenol diffusion in the distillate, yet the data obtained were rather contradictory. Such a phenomenon could be ascribed to the ultrasound-induced degradation of some phenolics, as outlined by relevant studies [21], but this hypothesis merits deeper investigation.

Aging with chestnut chips under SM yielded the most enriched sample, its $C_{\mathrm{TP}(s)}$ being $481.47 \mathrm{mg} \cdot \mathrm{GAE} \cdot \mathrm{L}^{-1}$. Treatment with oak chips under US also gave a highly enriched distillate $\left(C_{\mathrm{TP}(s)}=435.84 \mathrm{mg} \cdot \mathrm{GAE} \cdot \mathrm{L}^{-1}\right)$ but the use of cherry chips under US resulted in the least enriched one $\left(C_{\operatorname{TP}(s)}=128.63 \mathrm{mg} \cdot \mathrm{GAE} \cdot \mathrm{L}^{-1}\right)$. Data from the use of model wines and the same botanic species were in absolute concurrence, giving an order of richness in polyphenolic substances of chestnut $>$ oak $>$ acacia $>$ cherry [22]. This is also in agreement with previous findings on wine-like model systems, which showed the order of polyphenol enrichment to be oak > acacia > cherry, and also proportional to the amount of chips added [13].

\subsection{Antioxidant Activity}

Two complementary methods, the antiradical activity $\left(\mathrm{A}_{\mathrm{AR}}\right)$ and the reducing power $\left(\mathrm{P}_{\mathrm{R}}\right)$, were employed to evaluate the antioxidant activity of the aged distillate samples after 30 days of treatment. Samples aged with chestnut chips, treated either with SM or US, displayed statistically higher $P_{R}$ $(p<0.05)$, as opposed to the cherry samples, which both possessed significantly low $\mathrm{P}_{\mathrm{R}}$ (Figure 2 ). In the same fashion, the sample treated with chestnut chips under SM was the most active in free radical scavenging, but once again both samples aged with cherry chips were the least effective in this regard (Figure 3). In general, the antioxidant activity in distillates is attributed to polyphenols originating from the wood and it has been correlated with their concentration [23]. However, particular wood constituents may exert strong antioxidant effects [24,25], and therefore the antioxidant activity may be greatly affected by the species of the wood [13].

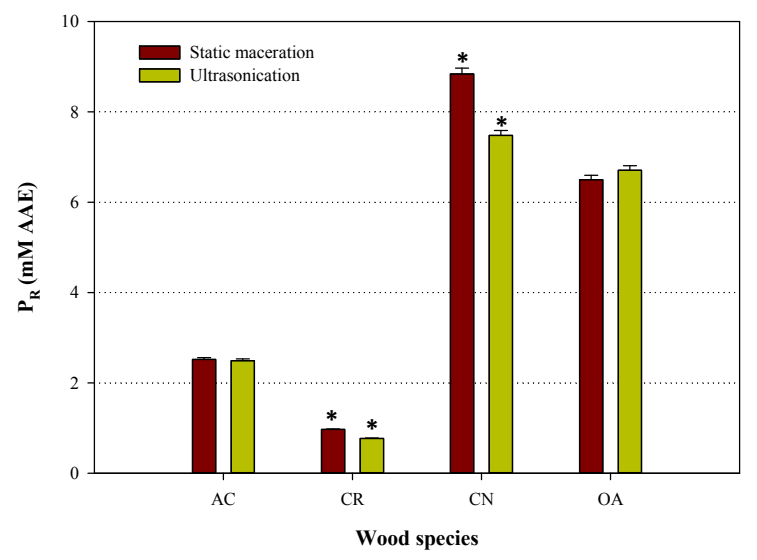

Figure 2. Comparative diagram showing $P_{R}$ of Tsipouro samples, aged with wooden chips under SM and US. Asterisk denotes statistically different values $(p<0.05)$. 


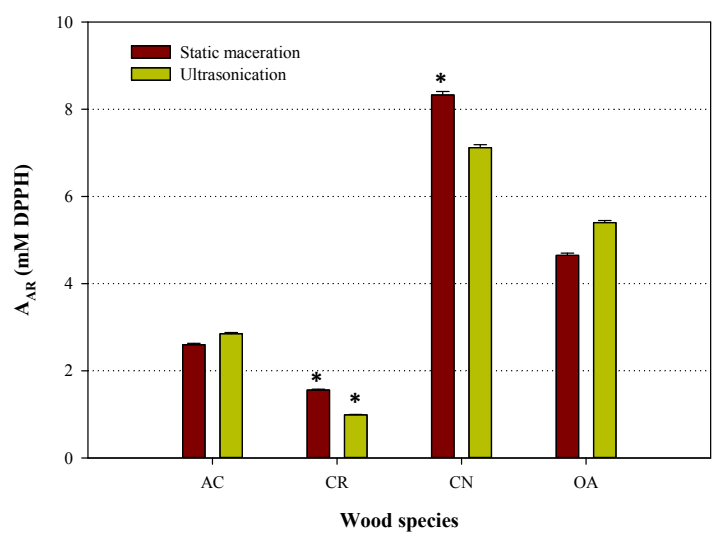

Figure 3. Comparative diagram showing $\mathrm{A}_{\mathrm{AR}}$ of Tsipouro samples, aged with wooden chips under SM and US. Asterisk denotes statistically different values $(p<0.05)$.

The data presented herein are in line with previous outcomes, which demonstrated wine distillates aged in chestnut barrels to display stronger antioxidant effects than those aged in oak barrels [26]. This activity was tightly linked with the hydrolyzable tannins, particularly ellagitannins (e.g., vescalagin, castalagin), occurring in chestnut wood. Such a phenomenon was also shown in hydroalcoholic model systems treated with non-toasted oak chips, where $C_{\mathrm{TP}}$ represented by hydrolyzable tannins was strongly correlated with $\mathrm{A}_{\mathrm{AR}}$ [17]. A further confirmation regarding the high antioxidant potency of hydrolyzable tannins came from the following examinations, which clearly showed that heavy toasting of oak chips resulted in extensive hydrolyzable tannin decomposition and a concomitant drastic decrease in both $\mathrm{A}_{\mathrm{AR}}$ and $\mathrm{P}_{\mathrm{R}}$ in wine model solutions [27].

The predominant polyphenolic forms in oak wood are hydrolyzable tannins $[26,28]$ and they possess strong antioxidant activity [29]. However, cherry tree heartwood has a different polyphenolic composition, containing flavonoids (mainly aromadendrin and naringenin) and low amounts of condensed tannins [30] and acacia wood contains various flavonoids [31]. In such a context and in order to have additional information regarding the polyphenolic composition of the aged samples, the total flavonoid concentration was also determined (Figure 4). Samples treated with acacia chips under both SM and US had the highest $C_{\mathrm{TFn}}$, which was virtually equal, amounting to 93.02 and $91.02 \mathrm{mg} \cdot \mathrm{RtE} \cdot \mathrm{L}^{-1}$, respectively. Both concentrations were significantly higher than those encountered in all the other samples $(p<0.05)$, but samples treated with cherry had notably low $C_{\mathrm{TFn}}$. Pairwise correlations showed that $C_{\mathrm{TP}}$ was highly correlated with both $\mathrm{A}_{\mathrm{AR}}$ and $\mathrm{P}_{\mathrm{R}}$, but $C_{\mathrm{TFn}}$ was correlated with neither (Table 2), thus providing evidence that the high antioxidant activity seen in chestnut-treated samples was exerted by components other than flavonoids.

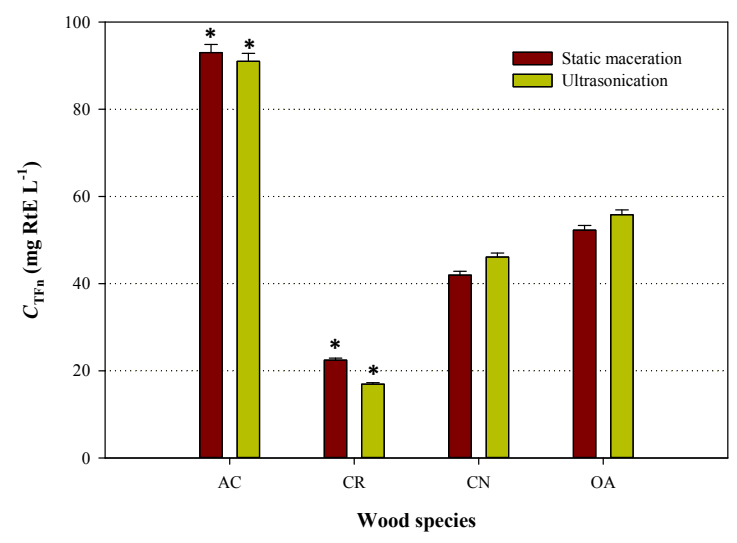

Figure 4. Comparative diagram showing $C_{\mathrm{TFn}}$ of Tsipouro samples, aged with wooden chips under SM and US. Asterisk denotes statistically different values $(p<0.05)$. 
Table 2. Statistical parameters estimated following pairwise correlations.

\begin{tabular}{cccccc}
\hline Variable & By Variable & Correlation & Lower 95\% & Upper 95\% & Significance \\
\hline $\mathrm{A}_{\mathrm{AR}}$ & $\mathrm{C}_{\mathrm{TP}}$ & 0.9475 & 0.7306 & 0.9907 & 0.0003 \\
$\mathrm{~A}_{\mathrm{AR}}$ & $\mathrm{C}_{\mathrm{TFn}}$ & 0.0357 & -0.6862 & 0.7222 & 0.9331 \\
$\mathrm{P}_{\mathrm{R}}$ & $\mathrm{C}_{\mathrm{TP}}$ & 0.9791 & 0.8851 & 0.9963 & $<0.0001$ \\
$\mathrm{P}_{\mathrm{R}}$ & $\mathrm{C}_{\mathrm{TFn}}$ & 0.0375 & -0.6853 & 0.7231 & 0.9297 \\
$\mathrm{P}_{\mathrm{R}}$ & $\mathrm{A}_{\mathrm{AR}}$ & 0.9741 & 0.8592 & 0.9955 & $<0.0001$ \\
\hline
\end{tabular}

\section{Conclusions}

This study is the first report on the accelerated aging of the traditional Greek grape marc distillate Tsipouro, using wooden chips from various botanical species. Tsipouro samples were subjected to the aging process under two different conditions, SM and US, to identify which technique could result in enhanced enrichment of the distillate in antioxidant polyphenols. The results obtained indicated that polyphenol release from the chips into the distillate followed first-order kinetics and that there was no statistical difference in the final total polyphenol concentration in samples treated with either SM or US after 30 days of aging. The determination of the antioxidant activity demonstrated that aging with chestnut chips may provide Tsipouro with particularly strong radical scavenging and reducing effects, highlighting its importance as a material that could be used to turn distillates into foods with functional (antioxidant) properties. Currently, work is in progress to assess the impact of the above aging techniques on the aromatic profile of Tsipouro.

Acknowledgments: The authors would like to thank the Department of Food Science \& Nutrition (University of the Aegean) for funding the research project.

Author Contributions: Theodora Taloumi carried out the experimental work; Dimitris P. Makris set up the experimental design, handled and processed raw data and wrote the paper.

Conflicts of Interest: The authors declare no conflict of interest.

\section{Nomenclature}

$\mathrm{A}_{\mathrm{AR}} \quad$ antiradical activity $(\mathrm{mM} \mathrm{DPPH})$

$C_{\mathrm{TP}} \quad$ total polyphenol concentration $\left(\mathrm{mg} \cdot \mathrm{GAE} \cdot \mathrm{L}^{-1}\right)$

$\mathrm{C}_{\mathrm{TFn}} \quad$ total flavonoid concentration $\left(\mathrm{mg} \cdot \mathrm{RtE} \cdot \mathrm{L}^{-1}\right)$

$\mathrm{P}_{\mathrm{R}} \quad$ reducing power (mM AAE)

\section{Abbreviations}

AAE ascorbic acid equivalents

$\mathrm{DPPH}^{\bullet} \quad$ 2,2-diphenyl-picrylhydrazyl radical

GAE gallic acid equivalents

RtE rutin (quercetin 3-O-rutinoside) equivalents

SM static maceration

TPTZ 2,4,6-tripyridyl-s-triazine

US ultrasonication

\section{References}

1. Mosedale, J.; Puech, J.-L. Wood maturation of distilled beverages. Trends Food Sci. Technol. 1998, 9, 95-101. [CrossRef]

2. Tao, Y.; García, J.F.; Sun, D.-W. Advances in wine aging technologies for enhancing wine quality and accelerating wine aging process. Crit. Rev. Food Sci. Nutr. 2014, 54, 817-835. [CrossRef] [PubMed]

3. Chang, A.C. Study of ultrasonic wave treatments for accelerating the aging process in a rice alcoholic beverage. Food Chem. 2005, 92, 337-342. [CrossRef]

4. Martín, J.F.G.; Sun, D.-W. Ultrasound and electric fields as novel techniques for assisting the wine aging process: The state-of-the-art research. Trends Food Sci. Technol. 2013, 33, 40-53. [CrossRef] 
5. Chemat, F.; Khan, M.K. Applications of ultrasound in food technology: Processing, preservation and extraction. Ultrason. Sonochem. 2011, 18, 813-835. [CrossRef] [PubMed]

6. Li, A.-N.; Li, S.; Zhang, Y.-J.; Xu, X.-R.; Chen, Y.-M.; Li, H.-B. Resources and biological activities of natural polyphenols. Nutrients 2014, 6, 6020-6047. [CrossRef] [PubMed]

7. Kana, K.; Kanellaki, M.; Papadimitriou, A.; Koutinas, A. Cause of and methods to reduce methanol content of Tsicoudia, Tsipouro and Ouzo. Int. J. Food Sci. Technol. 1991, 26, 241-247. [CrossRef]

8. Apostolopoulou, A.; Flouros, A.; Demertzis, P.; Akrida-Demertzi, K. Differences in concentration of principal volatile constituents in traditional Greek distillates. Food Control 2005, 16, 157-164. [CrossRef]

9. Geroyannaki, M.; Komaitis, M.E.; Stavrakas, D.E.; Polysiou, M.; Athanasopoulos, P.E.; Spanos, M. Evaluation of acetaldehyde and methanol in Greek traditional alcoholic beverages from varietal fermented grape pomaces (Vitis vinifera L.). Food Control 2007, 18, 988-995. [CrossRef]

10. Flouros, A.; Apostolopoulou, A.; Demertzis, P.; Akrida-Demertzi, K. Note: Influence of the packaging material on the major volatile compounds of tsipouro, a traditional Greek distillate. Food Sci. Technol. Int. 2003, 9, 371-378. [CrossRef]

11. Fotakis, C.; Christodouleas, D.; Kokkotou, K.; Zervou, M.; Zoumpoulakis, P.; Moulos, P.; Liouni, M.; Calokerinos, A. NMR metabolite profiling of Greek grape marc spirits. Food Chem. 2013, 138, 1837-1846. [CrossRef] [PubMed]

12. Fotakis, C.; Zervou, M. NMR metabolic fingerprinting and chemometrics driven authentication of Greek grape marc spirits. Food Chem. 2016, 196, 760-768. [CrossRef] [PubMed]

13. Psarra, C.; Gortzi, O.; Makris, D.P. Kinetics of polyphenol extraction from wood chips in wine model solutions: Effect of chip amount and botanical species. J. Inst. Brew. 2015, 121, 207-212. [CrossRef]

14. Manousaki, A.; Jancheva, M.; Grigorakis, S.; Makris, D.P. Extraction of antioxidant phenolics from agri-food waste biomass using a newly designed glycerol-based natural low-transition temperature mixture: A comparison with conventional eco-friendly solvents. Recycling 2016, 1, 194-204. [CrossRef]

15. Karakashov, B.; Grigorakis, S.; Loupassaki, S.; Makris, D.P. Optimisation of polyphenol extraction from Hypericum perforatum (St. John's Wort) using aqueous glycerol and response surface methodology. J. Appl. Res. Med. Aromat. Plants 2015, 2, 1-8. [CrossRef]

16. Shehata, E.; Grigorakis, S.; Loupassaki, S.; Makris, D.P. Extraction optimisation using water/glycerol for the efficient recovery of polyphenolic antioxidants from two Artemisia species. Sep. Purif. Technol. 2015, 149, 462-469. [CrossRef]

17. Karvela, E.; Makris, D.P.; Kefalas, P.; Moutounet, M. Extraction of phenolics in liquid model matrices containing oak chips: Kinetics, liquid chromatography-mass spectroscopy characterisation and association with in vitro antiradical activity. Food Chem. 2008, 110, 263-272. [CrossRef] [PubMed]

18. Tao, Y.; Zhang, Z.; Sun, D.-W. Experimental and modeling studies of ultrasound-assisted release of phenolics from oak chips into model wine. Ultrason. Sonochem. 2014, 21, 1839-1848. [CrossRef] [PubMed]

19. Makris, D.P. Kinetics of ultrasound-assisted flavonoid extraction from agri-food solid wastes using water/glycerol mixtures. Resources 2016, 5, 7. [CrossRef]

20. Chemat, F.; Rombaut, N.; Sicaire, A.-G.; Meullemiestre, A.; Fabiano-Tixier, A.-S.; Abert-Vian, M. Ultrasound assisted extraction of food and natural products. Mechanisms, techniques, combinations, protocols and applications: A review. Ultrason. Sonochem. 2017, 34, 540-560. [CrossRef] [PubMed]

21. Qiao, L.; Ye, X.; Sun, Y.; Ying, J.; Shen, Y.; Chen, J. Sonochemical effects on free phenolic acids under ultrasound treatment in a model system. Ultrason. Sonochem. 2013, 20, 1017-1025. [CrossRef] [PubMed]

22. Soares, B.; Garcia, R.; Freitas, A.M.C.; Cabrita, M.J. Phenolic compounds released from oak, cherry, chestnut and Robinia chips into a syntethic wine: Influence of toasting level. Ciênc. Téc. Vitiv. 2012, 27, 17-26.

23. Schwarz, M.; Rodríguez, M.; Martínez, C.; Bosquet, V.; Guillén, D.; Barroso, C.G. Antioxidant activity of Brandy de Jerez and other aged distillates, and correlation with their polyphenolic content. Food Chem. 2009, 116, 29-33. [CrossRef]

24. Alonso, A.M.; Castro, R.; Rodrıguez, M.C.; Guillén, D.A.; Barroso, C.G. Study of the antioxidant power of brandies and vinegars derived from Sherry wines and correlation with their content in polyphenols. Food Res. Int. 2004, 37, 715-721. [CrossRef]

25. Aoshima, H.; Tsunoue, H.; Koda, H.; Kiso, Y. Aging of whiskey increases 1,1-diphenyl-2-picrylhydrazyl radical scavenging activity. J. Agric. Food Chem. 2004, 52, 5240-5244. [CrossRef] [PubMed] 
26. Canas, S.; Casanova, V.; Belchior, A.P. Antioxidant activity and phenolic content of Portuguese wine aged brandies. J. Food Compos. Anal. 2008, 21, 626-633. [CrossRef]

27. Kanakaki, E.; Siderakou, D.; Kallithraka, S.; Kotseridis, Y.; Makris, D.P. Effect of the degree of toasting on the extraction pattern and profile of antioxidant polyphenols leached from oak chips in model wine systems. Eur. Food Res. Technol. 2015, 240, 1065-1074. [CrossRef]

28. Alañón, M.; Castro-Vázquez, L.; Díaz-Maroto, M.; Gordon, M.; Pérez-Coello, M. A study of the antioxidant capacity of oak wood used in wine aging and the correlation with polyphenol composition. Food Chem. 2011, 128, 997-1002. [CrossRef]

29. Vivas, N.; de Vivas Gaulejac, N.; Vitry, C.; Mouche, C.; Kahn, N.; Nonier-Bourden, M.F.; Absalon, C. Impact of ethanol content on the scavenging activities of oak wood $C$-glycosidic ellagitannins. Application to the evaluation of the nutritional status of spirits. J. Inst. Brew. 2013, 119, 116-125.

30. Sanz, M.; Cadahía, E.; Esteruelas, E.; Muñoz, A.N.M.; de Fernández Simón, B.G.; Hernández, T.; Estrella, I. Phenolic compounds in cherry (Prunus avium) heartwood with a view to their use in cooperage. J. Agric. Food Chem. 2010, 58, 4907-4914. [CrossRef] [PubMed]

31. Sanz, M.; de Simón, B.F.; Cadahía, E.; Esteruelas, E.; Muñoz, Á.M.; Hernández, M.T.; Estrella, I. Polyphenolic profile as a useful tool to identify the wood used in wine aging. Anal. Chim. Acta 2012, 732, 33-45. [CrossRef] [PubMed]

(C) 2017 by the authors; licensee MDPI, Basel, Switzerland. This article is an open access article distributed under the terms and conditions of the Creative Commons Attribution (CC-BY) license (http://creativecommons.org/licenses/by/4.0/). 